\title{
Hemoglobin-mediated Oxidant Damage to the Central Nervous System Requires Endogenous Ascorbate
}

\author{
Sayed M. H. Sadrzadeh and John W. Eaton \\ Department of Laboratory Medicine/Pathology and Dight Laboratories, University of Minnesota, Minneapolis, Minnesota 55455
}

\begin{abstract}
Hemorrhage within the central nervous system (CNS) may be associated with subsequent development of seizure states or paralysis. Prior investigations indicate that hemoglobin, released from extravasated erythrocytes, may be toxic to the CNS by promoting peroxidation of lipids and inhibition of Na,K-ATPase. These deleterious effects are blocked both in vitro and in vivo by the $\mathrm{Fe}^{3+}$ chelator, desferrioxamine, indicating the involvement of free iron derived from hemoglobin. We now report that the $\mathrm{Fe}^{2+}$ chelator, ferene, also inhibits methemoglobin- and ferric iron-mediated CNS lipid oxidation, reflecting the reduction of $\mathrm{Fe}^{3+}$ by some component of the CNS. This reduction is apparent in the accumulation of the highly chromophoric ferene: $\mathrm{Fe}^{2+}$ chelate after the addition of $\mathrm{Fe}^{3+}$ salts to supernatants of murine brain homogenates. Because large amounts of ascorbic acid occur in mammalian CNS, we suspected that this reducing substance might be responsible. Indeed, the peroxidative effects of hemoglobin and iron on murine brain are blocked by washing of CNS membranes or by preincubation of crude homogenates with ascorbate oxidase. Furthermore, the addition of ascorbate to washed CNS membranes fully restores hemoglobin/iron-driven peroxidation. We conclude that posthemorrhagic CNS dysfunction may stem from damaging redox reactions between hemoglobin iron, ascorbic acid, and oxidizable components of the nervous system.
\end{abstract}

\section{Introduction}

Integrity of the blood brain barrier is crucial to the normal functioning of the central nervous system (CNS), ${ }^{1}$ and cerebrospinal hemorrhage often is associated with subsequent development of seizure states $(1,2)$ and, perhaps, paralysis. The events leading to these sequelae are not fully understood but may involve inflammatory and oxidative reactions (3).

Earlier we reported several kindreds in which there was an association between familial idiopathic epilepsy and hypohap-

Dr. Sadrzadeh's present address is Department of Laboratory Medicine, University of Washington, Seattle, WA 98195.

Address reprint requests to Dr. John W. Eaton, Department of Laboratory Medicine/Pathology, Box 198 UMHC, University of Minnesota, Minneapolis, MN 55455.

Received for publication 12 November 1987 and in revised form 22 April 1988

1. Abbreviations used in this paper: CNS, central nervous system; TBARS, thiobarbituric acid reactive substances.

J. Clin. Invest.

(C) The American Society for Clinical Investigation, Inc. $0021-9738 / 88 / 11 / 1510 / 06 \$ 2.00$

Volume 82, November 1988, 1510-1515 toglobinemia (4). In these families, practically all the patients with seizure disorders had hypo- or an-haptoglobinemia, conditions that are very rare in the general population. Several unaffected individuals also had hypohaptoglobinemia. We hypothesized that very low levels of serum haptoglobin might impair the normal process of clearance of free hemoglobin from the CNS and that free hemoglobin might somehow lead to CNS dysfunction. In partial confirmation, the clearance of radiolabeled hemoglobin from the brains of hypohaptoglobinemic rodents is significantly slower than from the brains of normal animals (4). This defect in hemoglobin clearance can be corrected by prior complexation of hemoglobin with purified haptoglobin.

If allowed to remain within the CNS, hemoglobin may contribute to posttraumatic damage. Indeed, the intracortical instillation of both iron salts (5) and hemoglobin (6) causes the appearance of epileptiform seizures and/or electroencephalographic abnormalities. The damaging effect of ferruginous compounds on the CNS probably involves iron-driven oxidative reactions. In vitro, the addition of purified hemoglobin to homogenates of brain or spinal cord triggers the generation of large amounts of thiobarbituric acid reactive substances (TBARS) $(4,7)$. In vivo, the injection of hemoglobin into feline spinal cords causes inhibition of neuronal Na,K-ATPase, probably via oxidative reactions (7).

In the reactions of hemoglobin with CNS tissue, the proximate damaging species is probably free iron released from hemoglobin. The addition of submicromolar concentrations of the powerful iron chelator, desferrioxamine, will prevent hemoglobin/iron-driven peroxidation of lipids in CNS homogenates $(4,7)$. The very low concentrations of desferrioxamine required to block hemoglobin-induced lipid oxidation suggest that only small amounts of iron are released from hemoglobin and somehow cause peroxidation catalytically. We hypothesized that a necessary part of this catalysis would be the repetitive reduction of $\mathrm{Fe}^{3+}$ to $\mathrm{Fe}^{2+}$ by unknown substance(s) within the CNS. The present work was carried out to investigate further the interactions between CNS lipid and hemoglobin-derived iron and, most importantly, to define the reactions responsible for the catalysis of iron-driven oxidative destruction of CNS components.

\section{Methods}

All chemicals used were of reagent grade. Solutions containing iron were made up within a few minutes of use because of the well-known tendency of ferric iron to precipitate as insoluble salts.

Lipid peroxidation. Whole murine brain derived from male Swiss Webster 25-40-g animals was excised within $60 \mathrm{~s}$ after cervical dislocation. CNS tissue was washed thoroughly in ice-cold saline and ho-

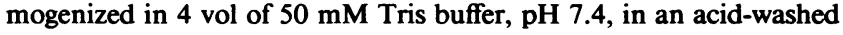
Potter-Elvehjem homogenizer. Incubations were performed at $25^{\circ} \mathrm{C}$ in a total volume of $1 \mathrm{ml}$ that contained $250 \mu$ diluted brain homogenate (protein concentration, $2.7 \mathrm{mg} / \mathrm{ml}$ ), $50 \mu \mathrm{M}$ purified hemoglobin (pre- 
pared according to Barksdale et al.) (8), $10 \mu \mathrm{M} \mathrm{FeSO}_{4}, 10 \mu \mathrm{M} \mathrm{FeCl}_{3}$, and $30 \mu \mathrm{M}$ desferrioxamine B or $250 \mu \mathrm{M}$ ferene [3-(2-pyridyl)-5,6bis(2-[5-furylsulfonic acid])-1,2,4-triazine]. After incubation, the concentrations of TBARS (aldehydic by-products of the peroxidation of unsaturated fatty acids) in 10\% TCA extracts was determined as previously described (9). Heating of the TCA/thiobarbituric acid extracts was carried out after centrifugation at $10,000 \mathrm{~g}$ to remove precipitated material. This step effectively blocked all artefactual generation of TBARS after the primary (experimental) incubation and obviated the need for addition of chelators such as desferrioxamine and antioxidants such as butylated hydroxytoluene (10).

Determination of reducing substance(s) in water-soluble fractions of brain. In some experiments we evaluated the possible importance of water-soluble reducing substances in causing iron-dependent lipid peroxidation. Homogenates were made from fresh murine brain as described above and centrifuged at $1,000 \mathrm{~g}$ for $10 \mathrm{~min}$. Supernatant was removed and the pellet washed three times with ice-cold saline in order to remove the water-soluble components. The pellet was then dispersed in ice-cold saline to yield a protein concentration of $\sim 2.7$ $\mathrm{mg} / \mathrm{ml}$. $\mathrm{FeCl}_{3}$ (final concentration $10 \mu \mathrm{M}$ ) was added to both washed and unseparated homogenates and incubated at $25^{\circ} \mathrm{C}$. After $1 \mathrm{~h}$ incubation, the extent of lipid peroxidation was measured as described above.

Characterization of brain-reducing substance(s). In further experiments we determined whether the water-soluble substances were heatstable and whether aqueous and membrane components could be reconstituted to permit "normal" iron-driven lipid peroxidation. Fresh brain homogenate was centrifuged at $10,000 \mathrm{~g}$ for $10 \mathrm{~min}$ and supernatant removed and saved. An aliquot of the supernatant was incubated at $100^{\circ} \mathrm{C}$ for $5 \mathrm{~min}$. The boiled or untreated supernatant was recombined with the washed membrane pellet and incubated at $25^{\circ} \mathrm{C}$ with added $\mathrm{FeCl}_{3}(10 \mu \mathrm{M})$. Untreated homogenate, also incubated with $\mathrm{FeCl}_{3}$, was used as control. After $1 \mathrm{~h}$ incubation, the concentration of TBARS was measured as described above. In some experiments, the reduction of $\mathrm{Fe}^{3+}$ to $\mathrm{Fe}^{2+}$ by the aqueous phase of murine brain homogenates was detected by the formation of an $\mathrm{Fe}^{2+} /$ ferene chelate. $\mathrm{Fe}^{3+}$ (final concentration $200 \mu \mathrm{M}$ ) was added to the supernatant of murine brain homogenates prepared as described above. The $\mathrm{Fe}^{2+}$ chelator, ferene, was also added to a final concentration of $1 \mathrm{mM}$. The appearance of $\mathrm{Fe}^{2+}$ resulting from the reduction of $\mathrm{Fe}^{3+}$ was followed spectrophotometrically at $594 \mathrm{~nm}$.

Ascorbate oxidase treatment. Fresh murine brain homogenate was prepared as described above and incubated with ascorbate oxidase (1 $\mathrm{U} / \mathrm{ml})$ for $15 \mathrm{~min}$ at $25^{\circ} \mathrm{C}, \mathrm{FeCl}_{3}(10 \mu \mathrm{M})$ or methemoglobin $(50 \mu \mathrm{M}$ as monomer) was added, and the mixture was incubated at $25^{\circ} \mathrm{C}$ for 60 min. At the beginning and end of the incubation period, TBARS concentration was measured as described above.

Ascorbic acid determination in brain homogenate. Fresh murine brain was homogenized in ice-cold saline (50\% wt/vol). To $1 \mathrm{ml}$ of whole brain homogenate, supernatant (boiled or untreated) or washed pellet, $1 \mathrm{ml}$ of $10 \%$ TCA was added, mixed well, and centrifuged at $1,000 \mathrm{~g}$ for $10 \mathrm{~min}$. The ascorbate concentration was determined on TCA extracts according to Day et al. (11).

\section{Results}

The addition of small amounts of purified methemoglobin to murine brain homogenate causes a large increase in the rate of formation of TBARS (Fig. 1). Added oxyhemoglobin has a similar, but slightly less pronounced, effect (Fig. 1).

The requirement for hemoglobin-derived iron is indicated by four lines of evidence. First, when heme-free globin is added in equivalent amounts to brain lipid homogenates, little or no lipid peroxidation ensues. Second, the addition of desferrioxamine B (30 $\mu \mathrm{M})$ completely blocks hemoglobin-driven brain lipid peroxidation (Fig. 1). Third, as discussed below, the ad-

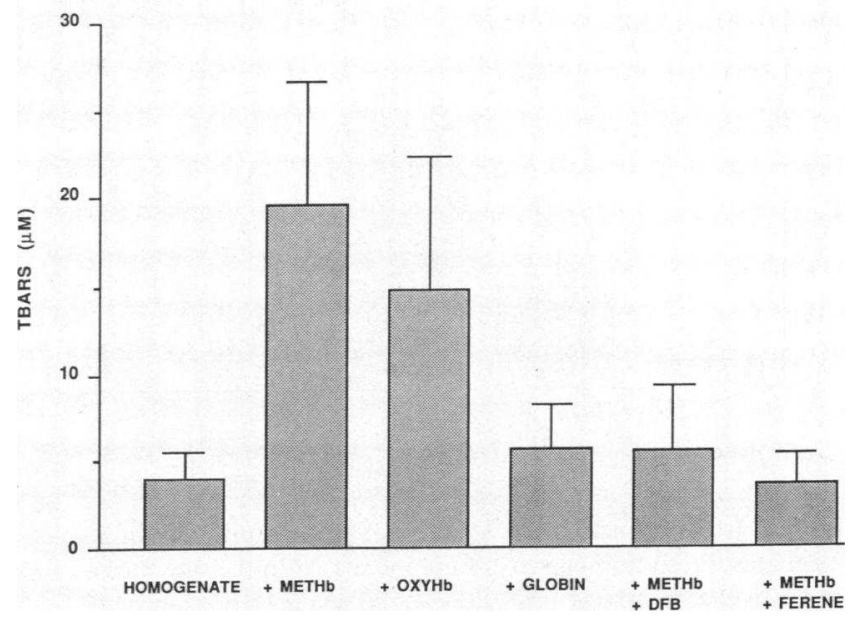

Figure 1. Hemoglobin-dependent peroxidation of crude murine brain homogenates. Fresh brain homogenates were incubated with methemoglobin or oxyhemoglobin $(50 \mu \mathrm{M}$, as monomer) or globin ( $50 \mu \mathrm{M}$, as monomer) in the presence or absence of desferrioxamine $\mathrm{B}(D F B, 30 \mu \mathrm{M})$ or ferene $(250 \mu \mathrm{M})$ as indicated. Incubations were carried out at $25^{\circ} \mathrm{C}$ for $60 \mathrm{~min}$. The concentration of TBARS was determined as described in Methods and the results expressed in molar terms calculated from a freshly prepared malondialdehyde standard (12). Results shown represent the mean \pm 1 SD of quadruplicate independent determinations. Mean values for methemoglobin and oxyhemoglobin differ significantly $(P<0.001$ in both cases) from homogenate (no addition). Similarly, generation of TBARS was significantly curtailed by addition of desferrioxamine and ferene vs. samples incubated with methemoglobin only $(P<0.01$ in both cases) ( $t$ test, two-tailed).

dition of free iron mimics the oxidative effect of hemoglobin on brain homogenates. Finally, we find that both oxy- and methemoglobin release measurable amounts of iron during incubations with murine brain homogenates. Hemoglobin was added to fresh brain homogenates (prepared as described under Methods). Any free iron formed was extracted by the addition of thiourea (final concentration, $15 \mathrm{mM}$ ) and sodium ascorbate (final concentration, $3 \mathrm{mM}$ ). After an additional minute of incubation with these agents, TCA was added (final concentration, 5\%) and iron was assayed spectrophotometrically in the deproteinized supernatant by reaction with ferene (13). $1 \mathrm{~h}$ after the addition of $50 \mu \mathrm{M}$ oxyhemoglobin (tetramer) to brain homogenate, there was a net increase of $5.0 \mu \mathrm{M}$ free iron. In a second experiment conducted under identical conditions, $4.5 \mu \mathrm{M}$ iron was generated $1 \mathrm{~h}$ after the addition of 50 $\mu \mathrm{M}$ methemoglobin. It should be noted that in both cases this is a minimal estimate; there is no certainty that all of the iron released was detectable with the extraction techniques employed.

As shown in Fig. 2, the addition of either ferric chloride or ferrous sulfate $(10 \mu \mathrm{M})$ reproduces the oxidative effect of hemoglobin. Although both oxidation states of iron will promote TBARS formation, under our conditions $\mathrm{Fe}^{2+}$ appears more effective than $\mathrm{Fe}^{3+}$ in mediating lipid oxidation (Fig. 2). However, regardless of whether $\mathrm{Fe}^{2+}$ or $\mathrm{Fe}^{3+}$ is added, the ferric iron chelator desferrioxamine almost totally prevents the irondriven formation of TBARS (Fig. 2), suggesting that added iron cycles between the two oxidation states. This is further supported by the observation that the addition of ferene, a chelator specific for ferrous iron, stops ferrous and ferric iron- 


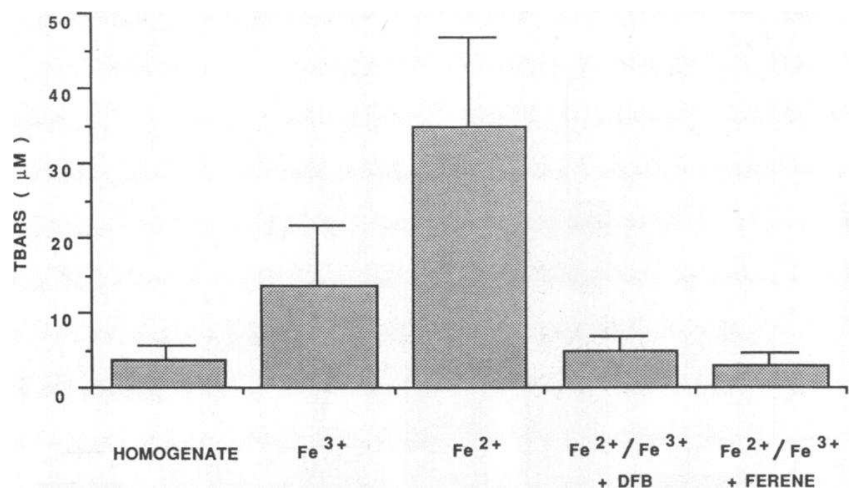

Figure 2. Iron-dependent peroxidation of crude murine brain homogenates. Fresh homogenates were incubated in the presence of 10 $\mu \mathrm{M} \mathrm{FeCl}\left(3\left(\mathrm{Fe}^{3+}\right), 10 \mu \mathrm{M} \mathrm{FeSO}\left(\mathrm{Fe}^{2+}\right), 30 \mu \mathrm{M}\right.$ desferrioxamine B $(D F B)$, or $250 \mu \mathrm{M}$ ferene. $\mathrm{Fe}^{2+} /^{3+}$ indicates that the results were identical in the presence of added desferrioxamine $B$ or ferene, regardless of which iron salts $\left(\mathrm{Fe}^{2+}\right.$ or $\left.\mathrm{Fe}^{3+}\right)$ were added. The results represent the mean \pm 1 SD of quadruplicate determinations. The addition of either $\mathrm{Fe}^{3+}$ or $\mathrm{Fe}^{2+}$ significantly enhanced TBARS formation $(P<0.001$ in both cases) and the chelators, desferrioxamine $B$ and ferene, suppressed $\mathrm{Fe}^{3+} / \mathrm{Fe}^{2+}$-dependent TBARS production $(P$ $<0.001$ in both cases) ( $t$ test, two-tailed).

mediated lipid peroxidation (Fig. 2). Interestingly, ferene also blocks methemoglobin-induced lipid peroxidation (Fig. 1). Because ferene will only chelate $\mathrm{Fe}^{2+}$, this observation indicates that the $\mathrm{Fe}^{3+}$ arising from methemoglobin must be converted to $\mathrm{Fe}^{2+}$ by substances in the CNS.

Further experiments provided direct support for the reduction of $\mathrm{Fe}^{3+}$ to $\mathrm{Fe}^{2+}$ within the CNS. When supernatants of murine brain homogenates are incubated with added $\mathrm{Fe}^{3+}$ and ferene, $\mathrm{Fe}^{3+}$ is rapidly reduced to $\mathrm{Fe}^{2+}$ as reflected by accumulation of the ferene/ $\mathrm{Fe}^{2+}$ chelate (Fig. 3). This clearly demonstrates the reduction of $\mathrm{Fe}^{3+}$ to $\mathrm{Fe}^{2+}$ by factors within brain homogenates.

The preceding experiments suggest that one or more unknown substances in the brain homogenate are responsible for the cycling of free iron. As a first step in identifying the reductive component(s), we prepared membrane-rich fractions from crude brain homogenate by washing the membranes three

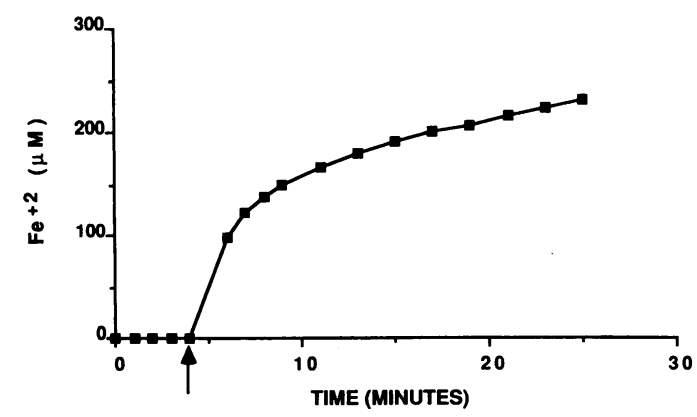

Figure 3. Reduction of $\mathrm{Fe}^{3+}$ to $\mathrm{Fe}^{2+}$ by a membrane-free supernatant of freshly prepared murine brain homogenate in the presence of 250 $\mu \mathrm{M} \mathrm{FeCl}{ }_{3}$ and $1 \mathrm{mM}$ ferene. The extent of $\mathrm{Fe}^{2+}$ formation was estimated by the appearance of a ferene/ $\mathrm{Fe}^{2+}$ chelate measured spectrophotometrically. The concentration of $\mathrm{Fe}^{2+}$ formed was calculated from the known absorbance coefficient of $\mathrm{Fe}^{2+} /$ ferene at $594 \mathrm{~nm}$ $\left(35.5 \mathrm{mM}^{-1} \mathrm{~cm}^{-1}\right)$ (13). Note that the amount of $\mathrm{Fe}^{2+}$ formed roughly equals the amount of $\mathrm{Fe}^{3+}$ added. times with multiple volumes of ice-cold saline to remove water soluble substances while leaving peroxidizable phospholipids. As shown in Table I, the addition of $\mathrm{Fe}^{3+}$ to washed membranes causes no TBARS formation, indicating that a soluble component of the reaction, presumably a reducing substance, is removed by washing. This conclusion is further strengthened by the observation that recombination of the primary supernatant of such homogenates with the washed membrane pellet fully restores the capacity of the brain homogenate lipids to peroxidize upon addition of exogenous $\mathrm{Fe}^{3+}$ (Table I). Further experiments indicate that the factor responsible is heat stable. Incubation of the supernatant of brain homogenate for $5 \mathrm{~min}$ at $100^{\circ} \mathrm{C}$ causes no diminution in its ability to mediate iron-driven lipid peroxidation (results not shown).

A number of water-soluble and heat-stable substances with the potential for reducing $\mathrm{Fe}^{3+}$ to $\mathrm{Fe}^{2+}$ may be present within the mammalian CNS. However, our attention was drawn to ascorbic acid. This reducing agent is present in the mammalian CNS at roughly 10 times the concentrations normally present in serum (14). To test the hypothesis that endogenous brain ascorbate is crucial in the above reactions, we added ascorbate oxidase $(1 \mathrm{U} / \mathrm{ml})$ to brain homogenate in the presence of either ferric chloride or methemoglobin. As shown in Table II, prior incubation of brain homogenate with ascorbate oxidase completely blocks methemoglobin/iron-mediated lipid peroxidation.

The ascorbic acid content of variously treated fractions of murine brain homogenate reflects their ability to undergo iron-driven peroxidation and supports the conclusion that endogenous ascorbate is the most important participant in TBARS formation in the presence of added ferruginous compounds. Untreated murine brain homogenate contains almost $0.5 \mathrm{mg}$ of ascorbate/g wet wt, and both untreated and boiled supernatants derived from such homogenates have similar concentrations of ascorbate. However, washed membranes have little ascorbate associated with them $(\sim 0.02 \mathrm{mg} / \mathrm{g}$ wet $w t)$. This is in accord with our finding that such washed membranes no longer exhibit iron-driven lipid peroxidation, whereas both untreated and boiled homogenate supernatants

Table I. Effect of Cytoplasmic Factor on $\mathrm{Fe}^{3+}$-mediated Lipid Peroxidation of Brain Homogenates*

\begin{tabular}{lcc}
\hline \multicolumn{1}{c}{ Sample } & TBARS & $n$ \\
\hline & $n$ mol/mg protein $\cdot h$ & \\
& $3.5 \pm 0.5$ & 6 \\
Untreated homogenate (with no addition) & $13.1 \pm 2.5^{\ddagger}$ & 6 \\
Untreated homogenate $+\mathrm{FeCl}_{3}(10 \mu M)$ & $1.9 \pm 0.1$ & 4 \\
Washed membranes (with no addition) & $2.4 \pm 0.14^{\S}$ & 4 \\
Washed membranes $+\mathrm{FeCl}_{3}(10 \mu M)$ & $3.8 \pm 0.4$ & 4 \\
Washed membranes + supernatant & & \\
Washed membranes + supernatant & $14.0 \pm 1.1^{\ddagger}$ & 4 \\
$\quad+\mathrm{FeCl}_{3}(10 \mu M)$ & &
\end{tabular}

* Incubations contained brain homogenates (1:4 brain:50 mM Tris, pH 7.4 [wt/vol]; untreated or washed) plus the additions indicated. Assays were carried out at $25^{\circ} \mathrm{C}$ for $1 \mathrm{~h}$.

${ }^{\S}$ Differ significantly from ${ }^{\ddagger}, P<0.001$ ( $t$ test, two-tailed).

"Supernatant derived from first $12,000 \mathrm{~g}$ centrifugation of homogenates added back to thrice washed membranes to reconstitute the original ratio of membrane protein/supernatant. 
Table II. Ascorbate Oxidase Blocks Methemoglobin and Ironmediated Peroxidation*

\begin{tabular}{lcr}
\hline \multicolumn{1}{c}{ Sample } & TBARS & $n$ \\
\hline & $n$ mol/mg protein $\cdot h$ & \\
& $2.93 \pm 1.6$ & 3 \\
Untreated homogenate (with no addition) & $22.0 \pm 4.2^{\ddagger}$ & 3 \\
Homogenate + metHb $(50 \mu M)$ & & \\
Homogenate + metHb $(50 \mu M)$ & $3.3 \pm 1.7^{8}$ & 3 \\
$\quad+$ ascorbate oxidase $(1 \mathrm{U} / \mathrm{ml})^{\| \prime}$ & $11.4 \pm 3.1^{\ddagger}$ & 4 \\
Homogenate $+\mathrm{FeCl}_{3}(10 \mu M)$ & & \\
Homogenate $+\mathrm{FeCl}_{3}(10 \mu M)$ & $2.4 \pm 1.5^{8}$ & 4 \\
+ ascorbate oxidase $(1 \mathrm{U} / \mathrm{ml})^{\| \prime}$ & & \\
&
\end{tabular}

* Brain homogenates (1:4 brain:50 mM Tris buffer, pH 7.4, [wt/vol]) were incubated with ascorbate oxidase $(1 \mathrm{U} / \mathrm{ml})$ where indicated, after which methemoglobin ( $50 \mu \mathrm{M}$, tetramer) and $\mathrm{FeCl}_{3}(10 \mu \mathrm{M})$ were added. Samples were then incubated at $25^{\circ} \mathrm{C}$ for $1 \mathrm{~h}$.

"These samples were pre-incubated with $1 \mathrm{U} / \mathrm{ml}$ of ascorbate oxidase for $20 \mathrm{~min}$ before addition of either methemoglobin or iron.

${ }^{8}$ Differ from ${ }^{\ddagger}, P<0.01$ ( $t$ test, two-tailed).

do support membrane peroxidation. Finally, the addition of exogenous ascorbate to washed membranes prepared from murine brain homogenate restores iron-driven lipid peroxidation. Although washed membranes accumulate no TBARS during $30 \mathrm{~min}$ incubation at $37^{\circ} \mathrm{C}$, the same membranes to which ascorbic acid has been added in amounts equal to those found in brain exhibit rapid lipid peroxidation similar to that of fresh brain homogenates (Table III).

\section{Discussion}

After traumatic or spontaneous hemorrhage, free hemoglobin may promote tissue injury. A most important instance of such hemoglobin-enhanced damage may be that which attends hemorrhage within the CNS. Removal from the CNS of free hemoglobin released by these extravascular red cells may present special difficulties. An additional circumstance that makes free hemoglobin particularly hazardous to the CNS is the presence of large amounts of readily oxidizable polyunsaturated fatty acids. The conjugation of free hemoglobin/iron

Table III. Ascorbic Acid Mediates Peroxidation of Murine Brain Lipids*

\begin{tabular}{lcr}
\hline \multicolumn{1}{c}{ Sample } & TBARS & $n$ \\
\hline & $n$ mol/mg protein & \\
& $3.9 \pm 0.8^{\ddagger}$ & 4 \\
Untreated homogenate & $2.1 \pm 0.5$ & 4 \\
Washed membranes $+\mathrm{FeCl}_{3}(10 \mu M)$ & & \\
Washed homogenate + ascorbate $(2 \mathrm{mM})$ & $30.0 \pm 4.8$ & 4 \\
$+\mathrm{FeCl}_{3}(10 \mu M)^{8}$ &
\end{tabular}

\footnotetext{
* Incubations contained 20\% (wt/vol) brain homogenates (untreated or washed) in $50 \mathrm{mM}$ Tris, pH 7.4, plus the additions indicated. Homogenates were centrifuged and the supernatant was boiled for 5 min and added to the washed pellets. The concentration of ascorbate added ( $2 \mathrm{mM})$ is slightly less than that originally found in brain homogenates $(2.5 \mathrm{mM})$. For a detailed description of the techniques, see Methods.

${ }^{8}$ Differ from ${ }^{\ddagger}, P<0.01$ ( $t$ test, two-tailed).
}

and polyunsaturated fatty acids within the brain or spinal column after hemorrhage may contribute to CNS damage and dysfunction arising after injury.

Hemoglobin-derived iron appears to be the proximate cause of peroxidative damage to CNS lipid and oxidant-sensitive enzymes such as $\mathrm{Na}, \mathrm{K}-\mathrm{ATPase}(4,7)$. The importance of iron compounds in the pathogenesis of CNS damage has been of interest to a number of investigators (e.g., 5, 6). Purified hemoglobin per se will mediate hydroxyl radical generation and participate in the peroxidation of fatty acids such as arachidonate (15). However, it appears that the most potent oxidative effects of free hemoglobin are due to small amounts of iron released during interactions of hemoglobin with lipids (4, 16). Free hemoglobin, injected into the spinal cords of anesthetized cats, causes substantial inhibition of neuronal $\mathrm{Na}, \mathrm{K}$ ATPase at the site of injection. The importance of free iron is indicated by the prevention of this damage by the simultaneous injection of desferrioxamine (7).

One puzzling aspect of these earlier observations was that very small amounts of hemoglobin-derived iron seem to act catalytically in promoting CNS lipid peroxidation. The nature of the reactions in which the iron was presumably cycling from the ferric to ferrous state was unknown. In the present investigations, we adduce evidence that endogenous CNS ascorbic acid, a reducing agent present in large amounts in nervous tissue, is a necessary partner in hemoglobin/iron-mediated oxidative damage to nervous tissue. This conclusion is supported by several lines of evidence. Removal of all water-soluble substances from brain homogenates causes the disappearance of iron-mediated lipid peroxidation, while the addition of membrane-free supernatants prepared from crude homogenates restores hemoglobin/iron-driven oxidation. The principle within these supernatants that sustains the peroxidative effects of iron is heat stable and survives boiling for at least $5 \mathrm{~min}$. However, this same principle is destroyed by the addition of ascorbate oxidase, indicating ascorbate as the major reducing substance involved. Indeed, the addition of concentrations of ascorbic acid equal to those normally occurring in crude brain homogenates restores the iron-driven peroxidation of washed neuronal membranes.

The precise reactions that ensue between hemoglobin iron, ascorbate, and unsaturated fatty acids are not yet fully elucidated. It is, however, clear that ascorbate is necessary for the reduction of $\mathrm{Fe}^{3+}$ to $\mathrm{Fe}^{2+}$. The latter, alone or in conjunction with $\mathrm{Fe}^{3+}(17,18)$, reacts readily with polyunsaturated fatty acids. This reaction of hemoglobin/iron with CNS fatty acids evidently requires repetitive redox cycling of the iron; either the $\mathrm{Fe}^{3+}$ iron chelator, desferrioxamine, or the $\mathrm{Fe}^{2+}$ iron chelator, ferene, will prevent hemoglobin/iron-driven CNS lipid peroxidation. In fact, after addition of $\mathrm{Fe}^{3+}$ to the supernatant of murine brain homogenates, substantial amounts of $\mathrm{Fe}^{2+}$ appear, as measured by formation of the ferene/ $\mathrm{Fe}^{2+}$ chelate. This observation unequivocally demonstrates that the brain contains substances, presumably ascorbate, effective in the reduction of $\mathrm{Fe}^{3+}$ to $\mathrm{Fe}^{2+}$. $\mathrm{Fe}^{2+}$ may be the more important form in the peroxidation of brain lipid as judged by its greater efficacy in mediating TBARS formation and its higher activity in the homolytic decomposition of lipid hydroperoxides (19). However, as shown schematically in Fig. 4, both $\mathrm{Fe}^{2+}$ and $\mathrm{Fe}^{3+}$ are requisite intermediates in the ascorbate-driven peroxidation of CNS lipid; complexation of either $\mathrm{Fe}^{2+}$ or $\mathrm{Fe}^{3+}$ by ferene and desferrioxamine, respectively, will totally abolish 


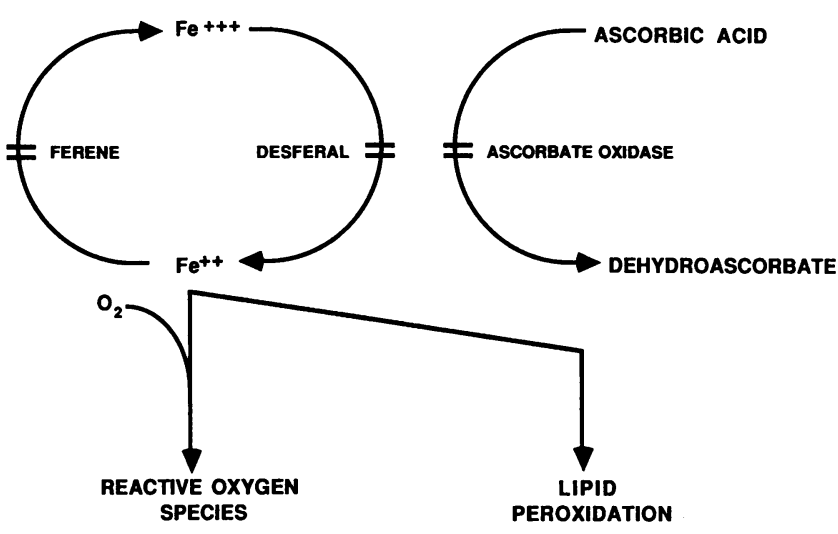

Figure 4. A schematic representation of iron oxidation/reduction cycling in hemoglobin/iron-mediated CNS damage. As indicated by the short horizontal pairs of bars, the process can be blocked by chelators of $\mathrm{Fe}^{2+}$ or $\mathrm{Fe}^{3+}$ or by specific enzymatic oxidation of ascorbic acid.

iron-driven brain lipid peroxidation. It is important to note that the requirement for both $\mathrm{Fe}^{2+}$ and $\mathrm{Fe}^{3+}$ may reflect the need for either redox cycling of the iron or, as proposed by Aust and co-workers $(17,18,20)$, the simultaneous presence of both forms of iron in achieving optimal rates of iron-driven peroxidation.

The pro-oxidant effects of exogenous ascorbic acid on various tissues have been reported by several groups $(21,22)$. Indeed, Sharma and Krishna Murti $(23,24)$ showed that endogenous ascorbate had a pro-oxidant effect on rat brain that was probably, in light of present results, mediated by endogenous CNS iron. Stimulation of lipid peroxidation by a combination of iron and low concentrations of ascorbate has been reported by others (20). Indeed, ascorbate added to brain homogenates (25) and synaptosomes (10) will accelerate $\mathrm{Fe}^{3+}$. dependent lipid peroxidation.

These earlier results, as well as those arising from our investigations, suggest a mechanism for hemoglobin/iron-mediated posthemorrhagic CNS damage involving the active participation of endogenous ascorbic acid as a reducing factor. That is, free methemoglobin derived from extravasated and lysed red cells spontaneously releases heme; the free heme subsequently degrades, releasing free $\mathrm{Fe}^{3+}(16)$. Ascorbic acid reduces $\mathrm{Fe}^{3+}$ to $\mathrm{Fe}^{2+}$, forming dehydroascorbate, which can be reduced to ascorbate by other reducing substances in the brain. Our experimental results indicate that this redox cycling of iron is associated with extensive peroxidation of brain lipids. In addition, ascorbate is an antioxidant (26) and is important in preventing the spontaneous oxidation of neurotransmitters such as catecholamines. Therefore, the redox cycling of hemoglobin/iron may not only facilitate the peroxidation of CNS lipids but also deplete an antioxidant necessary for storage of neurotransmitters.

Tissue damage mediated by free hemoglobin/iron is probably important in a variety of clinical circumstances. Foremost among these may be posttraumatic hemorrhage affecting the CNS. Hemorrhagic damage to the CNS is often associated with the development of functional disorders such as seizure states and paralysis. Our earlier observations suggest that impaired clearance of free hemoglobin from the CNS (secondary to hypohaptoglobinemia) may be an etiologic factor in the genesis of certain forms of familial and posttraumatic epilepsy
(4). Indeed, both in vitro and in vivo, hemoglobin/iron will cause substantial lipid oxidation and oxidant inhibition of enzymes important to neuronal function such as $\mathrm{Na}, \mathrm{K}-\mathrm{ATPase}$ $(4,7)$. In the present studies, we have defined the final element necessary for hemoglobin/iron-mediated damage to the CNS. Ascorbic acid, a reducing substance present in particularly large amounts in mammalian nervous tissue, is required for the redox cycling of iron and the associated peroxidation of brain lipid. It is paradoxical that ascorbic acid functions ordinarily to maintain oxidizable components of the brain in a reduced state. However, both CNS injury and even neuronal activity may cause release of ascorbate, whereupon this substance, rather than protecting against oxidation, may play a pivotal role in metal-catalyzed oxidative destruction of the CNS.

\section{Acknowledgments}

Partial support for this work was provided by the Graduate School of the University of Minnesota.

\section{References}

1. Gumnit, R. J. 1983. The Epilepsy Handbook. Raven Press, New York.

2. Loiseau, P., and P. Jallon. 1981. Post-traumatic epilepsy. Prog. Neurol. Surg. 10:323-343.

3. Means, E. E., and D. K. Anderson. 1983. Neurophagia by leukocytes in experimental spinal cord injury. J. Neuropathol. \& Exp. Neurol. 42:707-719.

4. Panter, S. S., S. M. H. Sadrzadeh, P. E. Hallaway, J. L. Haines, V. E. Anderson, and J. W. Eaton. 1985. Hypohaptoglobinemia: association with familial epilepsy. J. Exp. Med. 161:748-754.

5. Willmore, L. J., G. W. Sypert, J. B. Munson, and R. W. Hurd. 1978. Chronic focal epileptiform discharges induced by injection of iron into rat and cat cortex. Science (Wash. DC). 200:1501-1503.

6. Rosen, A. D., and N. V. Frumin. 1979. Focal epileptogenesis after intracortical hemoglobin injection. Exp. Neurol. 66:277-284.

7. Sadrzadeh, S. M. H., D. K. Anderson, S. S. Panter, P. E. Hallaway, and J. W. Eaton. 1987. Hemoglobin potentiates central nervous system damage. J. Clin. Invest. 79:662-664.

8. Barksdale, A. D., B. E. Hedlund, B. E. Hallaway, E. S. Benson, and $A$. Rosenberg. 1975. The binding of azide to human methemoglobin $A_{o}$. Error analysis for the interpolative and noninterpolative methods. Biochemistry. 14:2695-2699.

9. Beuge, J. A., and S. D. Aust. 1978. Microsomal lipid peroxidation. Methods Enzymol. 52:302-310.

10. Braughler, J. M., L. A. Duncan, and R. L. Chase. 1986. The involvement of iron in lipid peroxidation: importance of ferric to ferrous ratios in initiation. J. Biol. Chem. 261:10282-10289.

11. Day, B. R., D. R. Williams, and C. A. Marsh. 1979. A rapid manual method for routine assay for ascorbic acid in serum and plasma. Clin. Biochem. 12:22-26.

12. Waravdkar, V. S., and L. D. Saslaw. 1959. A sensitive colorimetric method for the estimation of 2-deoxy sugars with the use of malondialdehyde-thiobarbituric acid reaction. J. Biol. Chem. 234:1945-1950.

13. Artiss, J. D., S. Vinogradov, and B. Zak. 1981. Spectrophotometric study of several sensitive reagents for serum iron. Clin. Biochem. 14:311-316.

14. Hornig, D. 1975. Distribution of ascorbic acid, metabolites and analogues in man and animals. Ann. NY Acad. Sci. 258:103-115.

15. Sadrzadeh, S. M. H., E. Graf, S. S. Panter, P. E. Hallaway, and J. W. Eaton. 1984. Hemoglobin: a biologic Fenton reagent. J. Biol. Chem. 259:11354-11356. 
16. Hebbel, R. P., W. T. Morgan, J. W. Eaton, and B. E. Hedlund. 1988. Accelerated autoxidation and heme loss due to instability of sickle hemoglobin. Proc. Natl. Acad. Sci. USA. 85:237-241.

17. Minotti, G., and S. D. Aust. 1987. The requirement for ferric in the initiation of lipid peroxidation by ferrous and hydrogen peroxide. J. Biol. Chem. 262:1098-1104.

18. Bucher, J. R., M. Tien, and S. D. Aust. 1983. The requirement for ferric in the initiation of lipid peroxidation by chelated ferrous iron. Biochem. Biophys. Res. Commun. 111:777-784.

19. Aust, S. D., and B. A. Svingen. 1982. The role of iron in enzymatic lipid peroxidation. In Free Radicals in Biology. Vol. V. W. Pryor, editor. Academic Press, Inc., New York. 1-26.

20. Bucher, J. R., M. Tien, L. A. Morehouse, and S. D. Aust. 1983. Redox cycling and lipid peroxidation: the central role of iron chelates. Fundam. Appl. Toxicol. 3:222-226.
21. Ottolenghi, A. 1959. Interaction of ascorbic acid and mitochondrial lipids. Arch. Biochem. Biophys. 79:353-363.

22. Barber, A. A. 1966. Lipid peroxidation in rat tissue homogenates: interaction of iron and ascorbic acid as the normal catalytic mechanism. Lipids. 1:146-151.

23. Sharma, S. K., and C. R. Krishna Murti. 1968. Production of lipid peroxides by brain. J. Neurochem. 15:147-149.

24. Sharma, O. P., and C. R. Krishna Murti. 1976. Ascorbic acid: a naturally occurring mediator of lipid peroxide formation in rat brain. J. Neurochem. 27:299-301.

25. Zaleska, M. M., and R. A. Floyd. 1985. Regional lipid peroxidation in rat brain in vitro: possible role of endogenous iron. Neurochem. Res. 10:397-410.

26. Bodannes, R. S., and P. C. Chan. 1979. Ascorbic acid as a scavenger of singlet oxygen. FEBS (Fed. Eur. Biochem. Soc.) Lett. 105:195-196. 